\title{
MODULAR FORMS ASSOCIATED TO CLOSED GEODESICS AND ARITHMETIC APPLICATIONS
}

\author{
BY SVETLANA KATOK
}

\begin{abstract}
For any Fuchsian group of the first kind and any even weight greater than 2, we prove that the relative Poincaré series associated to closed geodesics generate the space of cusp forms of the given weight. Those series have very interesting geometrical and arithmetic properties. For arithmetic subgroups of $\mathrm{SL}_{2} \mathbf{R}$ (with or without cusps), our construction allows us to define two natural rational structures on the space of cusp forms.
\end{abstract}

Let $\Gamma$ be a Fuchsian group of the first kind, acting on the upper-half plane $\mathcal{H}$, i.e. a discrete subgroup of $\mathrm{SL}_{2} \mathbf{R}$ with $\operatorname{vol}(\Gamma \backslash \mathcal{H})<\infty$. For each integer $k \geq 2$ the space of cusp forms of weight $2 k$ on $\Gamma, S_{2 k}(\Gamma)$, is a finite-dimensional complex Hilbert space with respect to the Petersson scalar product

$$
\langle f, g\rangle=\iint_{\Gamma \backslash \mathcal{H}} f(z) \overline{g(z)}(\operatorname{Im} z)^{2 k} d V,
$$

where $d V=(d x d y) / y^{2}$ is the hyperbolic measure on $\mathcal{H}$ invariant with respect to the action of $\mathrm{SL}_{2} \mathbf{R}$.

Notations. $\gamma=\left(\begin{array}{ll}a & b \\ c & d\end{array}\right) \in \Gamma ; j(\gamma, z)=c z+d ;\left.f\right|_{2 k} \gamma=f(\gamma z) j(\gamma, z)^{-2 k}$.

The following proposition gives a general construction of cusp forms on $\Gamma$.

Proposition 1. Let $\Gamma_{0}$ be a subgroup of $\Gamma$ and $f(z)$ a function holomorphic on $\mathcal{H}$ satisfying:

(i) $\left.f\right|_{2 k} \gamma=f$ for all $\gamma \in \Gamma_{0}$.

(ii) $\iint_{\Gamma_{0} \backslash \mathcal{H}}|f(z)|(\operatorname{Im} z)^{k} d V<\infty$.

Then the relative Poincaré series

$$
F(z)=\sum_{\gamma \in \Gamma_{0} \backslash \Gamma}\left(\left.f\right|_{2 k} \gamma\right)(z)
$$

converges absolutely on $\mathcal{H}$, uniformly on compact sets, and $F(z)$ belongs to the space $S_{2 k}(\Gamma)$.

To each hyperbolic element $\gamma_{0}=\left(\begin{array}{ll}a & b \\ c & d\end{array}\right) \in \Gamma\left(\left|\operatorname{tr} \gamma_{0}\right|>2\right)$ we associate a quadratic function $Q_{\gamma_{0}}(z)=c z^{2}+(d-a) z-b$ which has its zeros in the two hyperbolic fixed points of $\gamma_{0}$ and satisfies $Q_{\gamma_{0}}\left(\gamma_{0} z\right)=Q_{\gamma_{0}}(z) \cdot j^{-2}\left(\gamma_{0}, z\right)$. Applying Proposition 1 with $\Gamma_{0}=\left\langle\gamma_{0}\right\rangle$ and $f(z)=c / Q_{\gamma_{0}}(z)^{k}$, we obtain a relative Poincaré series, which we denote by $\theta_{k, \gamma_{0}}$. Here $c$ is an appropriate normalizing constant. We have $\theta_{k, \gamma_{0}}=\theta_{k, \gamma_{1}}$ if $\gamma_{0}$ and $\gamma_{1}$ are conjugate in $\Gamma$, i.e. if they define the same closed geodesics on $\Gamma \backslash \mathcal{k}$. For each $f \in S_{2 k}(\Gamma)$ and

Received by the editors September 22, 1983 and, in revised form, December 7, 1983. 1980 Mathematics Subject Classification. Primary 10D12, 10D07, 30F35. 
hyperbolic $\gamma_{0} \in \Gamma$ the integral

$$
r_{k}\left(f, \gamma_{0}\right)=\int_{z_{0}}^{\gamma_{0} z_{0}} f(z) Q_{\gamma_{0}}^{k-1}(z) d z
$$

is called the period of $f$ over the closed geodesic associated to $\gamma_{0}$. The relative Poincaré series $\theta_{k, \gamma_{0}}$ have the following property: their scalar product with any $f(z) \in S_{2 k}(\Gamma)$ equals the period of $f(z)$ over the corresponding closed geodesic. The relative Poincaré series were previously studied for general $\Gamma$ by Petersson [6, 5], Hejhal [1], and Wolpert [8], and for $\mathrm{SL}_{2} \mathrm{Z}$ by Zagier [9], Kohnen [2], Kohnen and Zagier [3], and Kramer (thesis, University of Maryland, 1983).

THEOREM 1. (i) The relative Poincaré series $\left\{\theta_{k, \gamma_{0}}\right\}$ for primitive hyperbolic elements $\gamma_{0} \in \Gamma$ generate the whole space $S_{2 k}(\Gamma)$.

(ii) Suppose $\Gamma$ is symmetric, i.e.

$$
\gamma \in \Gamma \Rightarrow \gamma^{\prime}=\epsilon \gamma \epsilon \in \Gamma \quad\left(\epsilon=\left(\begin{array}{rr}
-1 & 0 \\
0 & 1
\end{array}\right)\right),
$$

and let

$$
\theta_{k, \gamma_{0}}^{+}=\frac{\theta_{k, \gamma_{0}}+\theta_{k, \gamma_{0}^{\prime}}}{2}, \quad \theta_{k, \gamma_{0}}^{-}=\frac{\theta_{k, \gamma_{0}}-\theta_{k, \gamma_{0}^{\prime}}}{2 i} .
$$

Then each of the families of cusp forms $\left\{\theta_{k, \gamma_{0}}^{+}\right\}$and $\left\{\theta_{k, \gamma_{0}}^{-}\right\}$generates $S_{2 k}(\Gamma)$.

In view of the correspondence between the scalar products and periods, Theorem 1 immediately follows from the following statement.

THEOREM 2. (i) If for $f \in S_{2 k}(\Gamma), r_{k}\left(f, \gamma_{0}\right)=0$ for all hyperbolic $\gamma_{0} \in \Gamma$, then $f=0$.

(ii) If $\Gamma$ is symmetric and $r_{k}\left(f, \gamma_{0}\right)-r_{k}\left(f, \gamma_{0}^{\prime}\right)=0$ or $r_{k}\left(f, \gamma_{0}\right)+r_{k}\left(f, \gamma_{0}^{\prime}\right)=0$ for all hyperbolic $\gamma_{0} \in \Gamma$, then $f=0$.

The proof of Theorem 2 goes as follows. Consider the tangent bundle $T(\mathcal{H})$ of the upper-half plane $H$ provided with the metric on each fiber induced by the hyperbolic metric on $\mathcal{H}$. Then the unit tangent bundle $S(\mathcal{H})$ may be parametrized by local coordinates $(z, \zeta)$ where $z \in \mathcal{H}, \varsigma \in \mathbf{C},|\varsigma|=\operatorname{Im}(z)$. Let $M=\Gamma \backslash \mathcal{H}$ and $S(M)$ be the unit tangent bundle over $M$ obtained from $S(\mathcal{H})$ by factorization modulo $\Gamma$. The second coordinate $\zeta$ may be regarded as a complex-valued function on $S(\mathcal{H}), \varsigma: S(\not{H}) \rightarrow$ C. To any cusp form $f(z) \in$ $S_{2 k}(\Gamma)$ we can associate the function $f(z) \zeta^{k}$, which is well defined on $S(M)$ and has the following property: its integral over any closed geodesic considered as a curve in $S(M)$ equals the period of $f(z)$ over the same closed geodesic. Then we consider closed geodesics in $S(M)$ as closed orbits of the geodesic flow on $S(M)$ and apply a result of Livčic [4], which implies that any differentiable function with zero integrals over all closed geodesics is in fact a derivative of another differentiable function in the direction of the geodesic flow. We then show that the function corresponding to a nonzero cusp form cannot be such a derivative. 
TheOREM 3. Let $\gamma_{0}$ and $\gamma_{1}$ be primitive hyperbolic elements. Then

$$
r_{k}\left(\theta_{k, \gamma_{0}}, \gamma_{1}\right)-r_{k}\left(\theta_{k, \gamma_{0}^{\prime}}, \gamma_{1}^{\prime}\right)=(\text { const }) \times \sum_{p \in\left[\gamma_{0}\right] \cap\left[\gamma_{1}\right]} \mu_{p} P_{k-1}\left(\cos \theta_{p}\right),
$$

where the summation is over all intersection points $p$ of two closed geodesics $\left[\gamma_{0}\right]$ and $\left[\gamma_{1}\right], \mu_{p}= \pm 1, \theta_{p}$ is the angle at $p$ between oriented geodesics $\left[\gamma_{0}\right]$ and $\left[\gamma_{1}\right], P_{k-1}$ is the $(k-1)$ th Legendre polynomial. Note that $P_{k-1}(-x)=$ $(-1)^{k-1} P_{k-1}(x)$ for all $k$.

A subgroup $\Gamma$ of $\mathrm{SL}_{2} \mathbf{R}$ is called arithmetic if it is obtained from a quaternion algebra $A / \mathbf{Q}$ which splits over $\mathbf{R}$ (see $[\mathbf{7}, \S 9.2]$ ).

THEOREM 4. If $\Gamma$ is an arithmetic subgroup of $\mathrm{SL}_{2} \mathbf{R}$, then $\left\langle\theta_{\gamma_{0}}^{+}, \theta_{\gamma_{1}}^{-}\right\rangle \in \mathbf{Q}$ for any two hyperbolic elements $\gamma_{0}$ and $\gamma_{1}$.

Theorem 1(ii) and Theorem 4 enable us to define two rational structures on $S_{2 k}(\Gamma)$ :

$$
\begin{aligned}
& S_{2 k}^{+}(\Gamma)=\left\{f \in S_{2 k}(\Gamma):\left\langle f, \theta_{k, \gamma_{0}}^{-}\right\rangle \in \mathbf{Q} \text { for all hyperbolic } \gamma_{0} \in \Gamma\right\}, \\
& S_{2 k}^{-}(\Gamma)=\left\{f \in S_{2 k}(\Gamma):\left\langle f, \theta_{k, \gamma_{0}}^{+}\right\rangle \in \mathbf{Q} \text { for all hyperbolic } \gamma_{0} \in \Gamma\right\} .
\end{aligned}
$$

REMARK. From the Eichler isomorphism between $S_{2 k}(\Gamma)$ and $H_{P}^{1}(\Gamma, X)$, the first cohomology group of $\Gamma$ with coefficients in some module $X$ (see [7, Chapter 8]), one can deduce the existence of a rational structure on $S_{2 k}(\Gamma)$ in the case when $\Gamma \subset M_{2}(\mathbf{Q})$. In the case of arithmetic subgroups of $\mathrm{SL}_{2} \mathbf{R}$ with compact $\Gamma \backslash \mathcal{H}$, the straightforward application of the Eichler isomorphism gives only an "algebraic" structure on $S_{2 k}(\Gamma)$.

\section{References}

1. D. Hejhal, Monodromy group and Poincaré series, Bull. Amer. Math. Soc. 84 (1978), 339-376.

2. W. Kohnen, Beziehungen zwischen Modulformen halbganzen Gewichts und Modulformen ganzen Gewichts, Bonner Math. Schriften, 131, Bonn, 1981.

3. W. Kohnen and D. Zagier, Modular forms with rational periods, Proc. Durham Sympos. on Modular Forms, Ellis Horwood., 1984.

4. A. N. Liv xic, Some homology properties of U-systems, Mat. Zametki 10 (1971), 555-564; English transl., Math. Notes 10 (1971), 758-763.

5. H. Petersson, Zur analytischen Theorie der Grenzkreisgruppen. V, Math. Z. 44 (1939), $127-155$.

6. __ Einheitliche Begründung der Vollständig keitssätze für die Poincaréschen Reihen, Abh. Math. Sem. Univ. Hamburg 14 (1941), 22-60.

7. G. Shimura, Introduction to the arithmetic theory of automorphic functions, Iwanami Shoten, Tokyo and Princeton Univ. Press, Princeton, N. J., 1971.

8. S. Wolpert, The Fenchel-Nielsen deformation, Ann. of Math. (2) 115 (1982), 501-528. $1-46$.

9. D. Zagier, Modular forms associated to real quadratic fields, Invent. Math. 30 (1975),

Department of Mathematics, University of California, Berkeley, CaliFORNIA 94720 